\title{
Atención Odontológica Durante el Estado de Alarma COVID-19 en el Hospital Comunitario de Achao, Chile: Un Estudio Observacional
}

\author{
Dental Care During the COVID-19 State of Emergency at the \\ Community Hospital of Achao, Chile: An Observational Study
}

Camila Francisca Barrientos García

BARRIENTOS, G. C. F. Atención Odontológica durante el estado de alarma COVID-19 en el Hospital Comunitario de Achao, Chile: Un estudio observacional. Int. J. Odontostomat., 15(3):583-585, 2021.

RESUMEN: Conocer la prevalencia de las patologias odontológicas diagnósticas en la población adscrita en el Hospital comunitario de Achao ubicado en Achao, comuna de Quinchao, de mayo a diciembre de 2020. Se realizó un estudio observacional descriptivo a traves de la revisión de la totalidad de atenciones odontológicas realizadas en el Hospital comunitario de Achao, durante mayo a diciembre de 2020, registrando el sexo, la edad y el diagnóstico clínico según el motivo de consulta del paciente, realizado siempre por el mismo observador. Se revisaron 262 notificaciones que cumplieron con los criterios de inclusión. El sexo femenino fue el que consultó con mayor frecuencia. La edad del $76 \%$ de la población fue entre 19 a 60 años. 159 notificaciones correspondieron a urgencias odontológicas ambulatorias GES, siendo los diagnósticos más prevalentes; Periodontitis apical sintomática y pulpitis. Por otra parte, se observaron consultas por desalojo de obturaciones y piezas con periodontits crónica severa. La pandemia ha generado una reorganización considerable en lo que respecta a las atenciones dentales, la literatura muestra que es recomendable suspender las atenciones electivas y restringuirse a la atención de urgencias, de pacientes críticos y procedimientos que no puedan ser postergados; En estos casos, se deben adoptar todas las medidas de protección antes, durante y despues de la atención dental.

PALABRAS CLAVE: atención odontológica, pandemia, COVID-19

\section{INTRODUCCIÓN}

El 30 de enero 2020, la OMS declara que el brote de COVID-19 constituye una Emergencia de Salud Pública de importancia Internacional, el 11 de marzo 2020 se declara pandemia global, dada la alta propagación del virus a nivel mundial. En Chile, el Ministerio de Salud (2020) confirmó el primer caso el 3 de marzo de 2020 y a partir de éste, el brote epidémico se expandio hasta alcanzar las dieciséis regiones del país.. Castillo et al. (2020) menciona que la pandemia por COVID-19 ha representado un desafío para los profesionales y los sistemas de salud de todo el mundo debido a su alta tasa de contagio y propagación. Entre todos, la profesión odontológica es una de las que presenta mayor riesgo de contagio, dada la proximidad con pacientes sintomáticos 0 asintomáticos, la exposición constante a fluidos cor- porales (sangre y saliva) y la potencial interacción con aerosoles generados durante los procedimientos odontológicos. Como medida sanitaria durante el periodo de transmición comunitaria del virus se suspendió todas las atenciones odontológicas electivas y se atendieron solo urgencias. Las urgencias dentales en Chile están dentro de las Garantías Explícitas en Salud (GES) según la Guía Clínica GES: Urgencias Odontológicas Ambulatorias, que se encuentra centrada en el manejo de condiciones que requieren de atención inmediata para aliviar dolor severo y/o riesgo de infección. El objetivo de este estudio es conocer la prevalencia de las patologias diagnósticadas en la población adscrita en el Hospital comunitario de Achao ubicado en Achao, comuna de Quinchao, durante mayo de 2020 hasta diciembre de 2020. 


\section{MATERIAL Y MÉTODO}

Se realizó un estudio observacional descriptivo para conocer las patologias odontológicas más prevalentes presentadas en la población adscrita en el Hospital comunitario de Achao durante el periodo de pandemia desde mayo a diciembre de 2020. Dicho estudio constó con la aprobación de Jefe Odontológico y Director del establecimiento. Para la obtención de los datos, se revisaron la totalidad de las notificaciones odontológicas realizadas durante ese periodo de tiempo. Se implementó un triage de urgencia odontológica, donde el paciente asistió a urgencia general del Hospital de Achao indicando su motivo de consulta, se controlaba signos vitales (incluyendo $\mathrm{T}^{\circ}$ ) y aplicación de un tamizaje de sospecha de COVID-19; Si no presentaban signos de alarma, se realizaba llamada telefónica a Odontólogo antes de realizar la atención clínica. En la recopilación de datos se registró el sexo, la edad y el diagnóstico clínico según el motivo de consulta del paciente, realizado siempre por el mismo odontólogo. La búsqueda fue realizada por un solo observador hasta obtener la totalidad de los registros. Los datos obtenidos, se tabularon en una planilla de Microsoft Office Excel 2013 y los resultados se analizaron mediante estadística descriptiva considerando las variables demográficas de la población donde se incluyó sexo y edad con el programa IBM SPSS STATISTICS Versión 22.

\section{RESULTADOS}

Se revisaron 262 atenciones que cumplieron con los criterios de inclusión. El $56 \%$ de los usuarios atendidos fue de sexo femenino(148 usuarios) y un $44 \%$ masculino (114 usuarios). El $76 \%$ de los usuarios se encontraba en el rango de 19 a 60 años de edad, un $16 \%$ de 61 a 80 años y un $8 \%$ menores de 18 años.

Un $60 \%$ de los diagnósticos realizados correspondieron a urgencias odontológicas ambulatorias GES; de ellas, la más prevalente fue Periodontitis apical sintomática con76 notificaciones, seguido de Pulpitis con 36 notificaciones. De los pacientes que presentaron Absceso de espacios faciales, solo un usuario cumplio con criterios de hospitalización. Por otra parte, tambien se observaron notificaciones de patologías no incluidas enUOA GES; de ellas,se observó 41 notificaciones por desalojo de obturaciones y 22 notificaciones por piezas dentarias con periodontitis crónica severa. Todas las consultas que no correspondían a las antes mecionadas se registraron en la categoría otros (caries, resto radicular, sensibilidad, recambio dentario, gingivitis crónica, malposición dentaria y consultas protésicas). Se detallan los resultados en la Tabla I.

Tabla I. Número y porcentaje de notificaciones según diagnóstico.

\begin{tabular}{lcc}
\hline & Frecuencia & $\%$ \\
\hline Perico ronaritis & 8 & 3,0 \\
Pulpitis & 43 & 16,4 \\
Periodontitis Apical Sintomática & 76 & 29,2 \\
Adaa & 6 & 2,2 \\
Absceso Submucoso & 13 & 5,0 \\
Absceso Espacio Facial & 12 & 4,6 \\
TDA & 1 & 0,4 \\
Alveolitis & 7 & 2,7 \\
Periodontitis Crónica & 22 & 8,4 \\
Desalojo de Obturación & 39 & 14,8 \\
Otros & 35 & 13,3 \\
Total & 262 & 100 \\
\hline
\end{tabular}

\section{DISCUSIÓN}

La reorganización oportuna de los servicios de atención dental ha sido un desafío durante la pandemia. El manejo temprano de las urgencias dentales agudas es importante para evitar que los pacientes acudan a los servicios de emergencias hospitalarios. Una preocupación considerable señalada por Dave et al. (2020) es que, con la suspensión de la atención dental de rutina, más pacientes de lo habitual podrían llegar a necesitar ingreso para tratamiento de infecciones dentales que podrían llegar a amenazar las vías respiratorias y necesitar cuidados intensivos.

En el estudio de Ramírez et al. (2021) realizado en España, se observó que la frecuencia de asistencia de los usuarios a los centros de salud disminuyó por miedo a acudir a los centros de salud de emergencia por la pandemia de COVID-19, lo que provocó un aumento en la prevalencia de la automedicación con analgésicos y AINES. También, cabe señalar que sus resultados coinciden con los obtenidos en el presente estudio respecto a los datos demográficos y diagnósticos más notificados. 


\section{CONCLUSIONES}

La pandemia ha generado una reorganización considerable en lo que respecta a las atenciones dentales, la literatura muestra que es recomendable suspender las atenciones electivas y restringuirse a las atenciones de urgencias odontologicas ambulatorias, de pacientes críticos y procedimientos que no puedan ser postergados. En el caso de la atención de urgencias, todo paciente debe ser considerado potencialmente contaminante y por lo tanto se deben adoptar todas las medidas de protección antes, durante y despues de la atención dental.

BARRIENTOS, G. C. F. Dental care during the COVID-19 state of emergency at the Community Hospital of Achao, Chile: An observational study. Int. J. Odontostomat., 15(3):583-585, 2021.

ABSTRACT: The objective of this study was to know the prevalence of diagnostic dental pathologies in the population assigned to the Achao Community Hospital located in Achao, Quinchao commune, from May 2020 to December 2020. A descriptive observational study was carried out through the review of all dental care performed at the Achao Community Hospital, during May to December 2020 , recording sex, age and clinical diagnosis according to the reason for consultation of the patient, always carried out by the same observer. 262 notifications that met the inclusion criteria were reviewed. It was noted that females consulted most frequently. The age of $76 \%$ of the population was between 19 to 60 years. 159 notifications corresponded to GES outpatient dental emergencies, being the most prevalent diagnoses; Symptomatic apical periodontitis and pulpitis. On the other hand, consultations were observed due to the dislodgement of fillings and pieces with severe chronic periodontitis. The pandemic has generated a considerable reorganization with regard to dental care, the literature shows that it is advisable to suspend elective care and restrict it to outpatient dental emergencies, critical patients and procedures that cannot be postponed; in these cases, all protective measures must be taken before, during and after dental care.

KEY WORDS: dental care, pandemic, COVID-19.
Ministerio de Salud. Informe Epidemiológico COVID-19. Enfermedad Producida por el Virus SARS-CoV-2. Chile 01-04-2020. Santiago de Chile, Departamento de Epidemiología, Ministerio de Salud, Gobierno de Chile, 2020. Disponible en: https:// www.minsal.cl/wp-content/uploads/2020/04/segundo-informeepidemiologico-covid.pdf

Ramírez, J.; Varela, L.; Gómez, D.; Giovannini, G.; Romero, M. \& Gómez, R. Management of odonto-stomatological emergencies during the COVID-19 alarm state in dental clinics in the Autonomous Community of Madrid (CAM), Spain: An observational study. Med. Oral Patol. Oral Cir. Bucal, 26(1):e1147, 2021.

Dirección para correspondencia:

Camila Barrientos García

Coñab SN

Achao

CHILE

E-mail: camilabarrientosgarcia@gmail.com

\section{REFERENCIAS BIBLIOGRÁFICAS}

Castillo, M.; Serpa, X. \& Wilches J. La odontología frente a la pandemia por covid-19: Medidas y prácticas a implementar. Rev. Esp. Salud Pública, 94:e1-4. 2020.

Dave, M., Seoudi, N. \& Coulthard, P. Urgent dental care for patients during the COVID-19 pandemic. Lancet, 395(10232):1257, 2020. 